\title{
DESARROLLO DE UN PROCESO DE MORFOGÉNESIS BASADO EN COMPUTACIÓN HEURÍSTICA MULTI-OBJETIVA PARA UNA ESTRUCTURA RETICULAR DE CUBIERTA*
}

Leonardo Moreno de Luca**

Universidad Industrial de Santander, Colombia

Oscar Javier Begambre Carrillo****

Universidad Industrial de Santander, Colombia

Recibido: 5 agosto 2013

Aprobado: 27 septiembre 2013
Geometría de entrada necesaria para Grasshopper.

Elaboración de los autores.

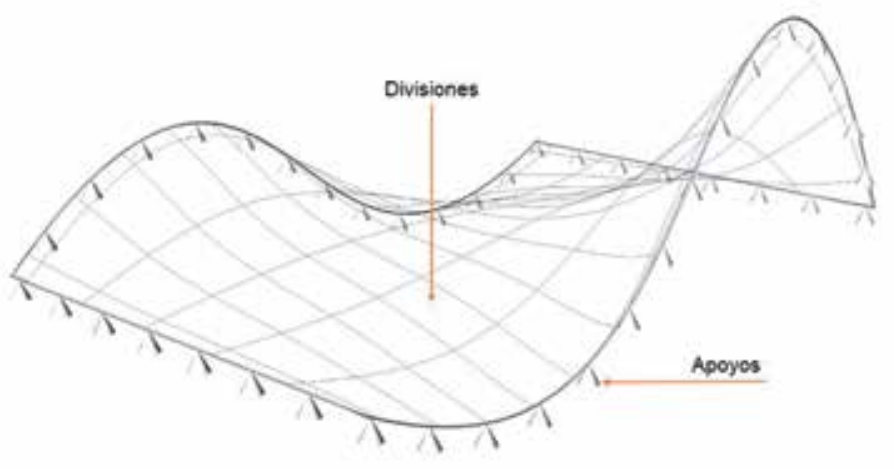

\section{RESUMEN}

En este artículo se presenta el desarrollo de un proceso de morfogénesis, el cual pretende encontrar la configuración geométrica óptima para una estructura reticular de cubierta. El procedimiento propuesto está basado en un algoritmo heurístico, el Unified Particle Swarm Optimization, UPSO (Parsopoulos, K. y Vrahatis, M.N., 2004), en el cual se tienen como objetivos la minimización del peso de la estructura y su energía de deformación. De esta manera, se busca obtener, simultáneamente, la topología estructural más liviana y la que presenta los menores desplazamientos. En los problemas de prueba, el proceso de optimización se ejecutó con distintas combinaciones de pesos para lograr una aproximación al frente de Pareto, donde se puede seleccionar para el diseño estructural definitivo, la cubierta estéticamente más agradable, conservando el alto rendimiento que caracteriza a las soluciones presentes en dicho grupo. De los resultados obtenidos, se concluyó que el procedimiento llevado a cabo es capaz de generar y optimizar la configuración geométrica de estructuras reticulares de cubierta, obteniendo así, reducciones (en promedio) en el peso del orden de $40 \%$, y en la energía de deformación de $60 \%$, en comparación con las estructuras iniciales generadas aleatoriamente.

\section{PALABRAS CLAVE}

Computación Heurística, Optimización Multiobjetiva, Estructuras Reticulares, Morfogénesis, Diseño Conceptual Estructural. 


\section{DEVELOPMENT OF A MULTI-OBJECTIVE- HEURISTIC-COMPUTING-BASED \\ MORPHOGENETIC PROCESS FOR A GRID ROOF STRUCTURE}

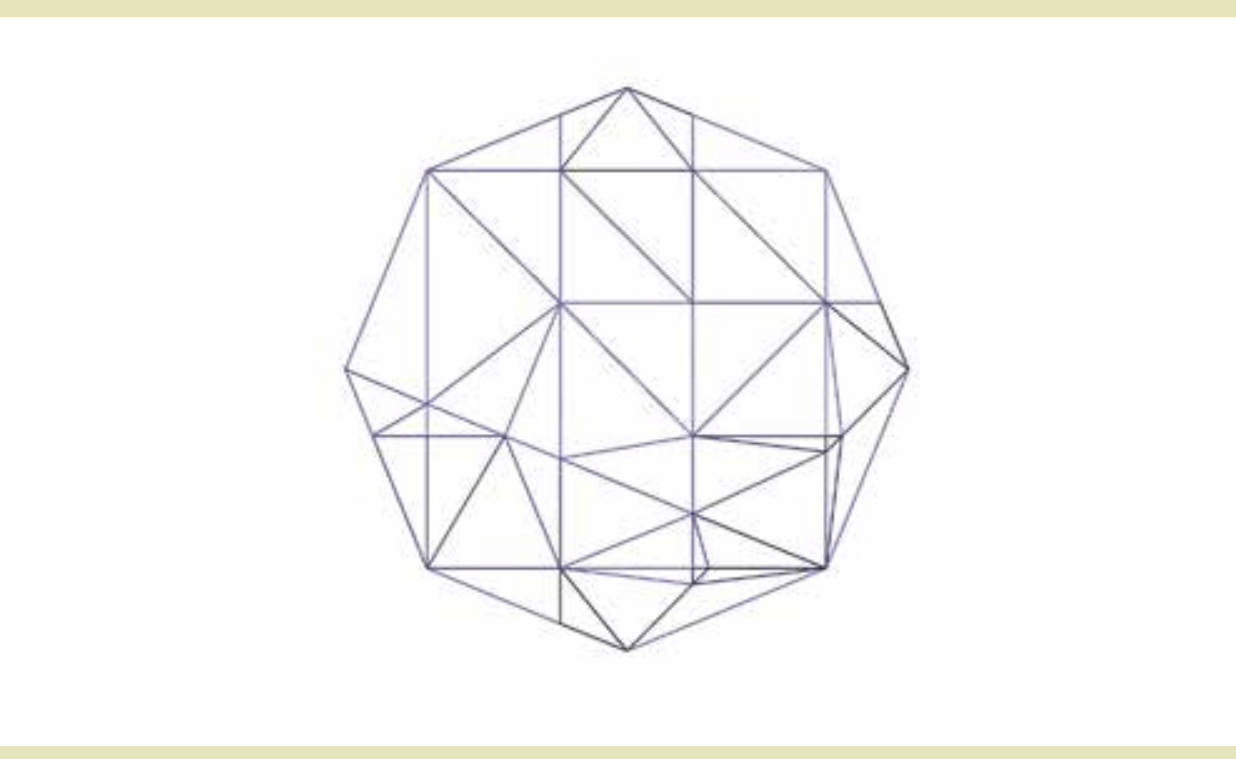

\section{ABSTRACT}

In this manuscript we develop a morphogenetic process, which aims to find the optimal geometric conFiguration for a grid roof structure. The proposed procedure is based on the Unified Particle Swarm Optimization, UPSO, (Parsopoulos, K. y Vrahatis, M.N. 2004), approach, i.e. an heuristic algorithm. The objectives taken into account within the algorithm were the weight and the strain energy minimization. In this context, it is possible to obtain the lightest structural topology and, simultaneously, the one with minimum strains, as the final result. In order to reach an approximation to the Pareto front, the optimization process was applied with different weight combinations; thus, the most aesthetically pleasant solution could be selected to develop the whole structural design process, while maintaining the high performance that characterize the solutions within such group. From the results obtained, it can be concluded that the proposed procedure is able to generate and optimize the geometrical conFiguration of grid roof structures, reaching weight decreases up to $40 \%$ (average), while regarding the strain energy this value went up until $60 \%$ (average); these results were obtained making a comparison with the randomly generated initial structures.

\section{KEYWORDS}

Heuristic Computation, Multi-objective Optimization, Grid Structures, Morphogenesis, Structural Conceptual Design.
Planta de estructura reticular de cubierta. Elaboración de los autores.

en el área de optimización estructura y computación evolutiva. Actualmente es miembro del grupo de investigación INME-UIS donde desarrolla su trabajo sobre "Aplicaciones de Meta Heurísticas en Problemas de Ingeniería Civil y Arquitectura. Es referee de importantes revistas internacionales $y$ ha publicado diversos trabajos sobre detección de daño en estructuras, optimización topológica y algoritmos para computación evolutiva.

E-mail: ojbegam@uis.edu.co 


\section{INTRODUCCIÓN}

Una de las principales metas en el diseño conceptual de una estructura reticular de cubierta, es lograr obtener una configuración geométrica eficiente (desde el punto de vista estructural), liviana (desde el punto de vista económico y estructural), y estéticamente agradable. Los arquitectos e ingenieros que han logrado conseguir esta condición lo han hecho, por lo general, guiados por la intuición intrínseca que se ha venido desarrollando en ellos luego de una gran experiencia en el tema, respaldada por un amplio bagaje de conocimiento. Por ejemplo, personajes como Buckminster Fuller, Frei Otto y Antonio Gaudí, lograban, por medio de su intuición, sabiduría e ingenio, proponer formas estructurales totalmente eficientes y con un alto valor estético.

La intención de esta investigación es intentar acercarse a ese tipo de topologías estructurales por medio de la computación heurística, la cual servirá como fundamento de un proceso morfogenético de optimización, enfocado en objetivos de rendimiento estructural. La forma obtenida será entonces resultado de la evolución de distintas posibilidades de solución, en vez de surgir de un proceso netamente manual, en donde la vasta experiencia es condición ineludible para lograr un resultado que se acerque a una condición óptima, sin siquiera poder comprobarla. Algunos trabajos de investigación en el tema son los presentados por Pugnale y Sassone (2007) y Byrne Fenton, Hemberg, McDermott, O'Neil, Shotton y Nally (20I I).

El presente trabajo está organizado de la siguiente manera: inicialmente se encuentra la descripción del problema; posteriormente se describe el proceso de optimización multiobjetiva que fue llevado a cabo (proceso de morfogénesis) y se da paso a la presentación de la aplicación del proceso de optimización propuesto en dos problemas de prueba, incluyendo los resultados obtenidos para finalmente, plantear conclusiones.

\section{DESCRIPCIÓN DEL PROBLEMA}

El problema de optimización es el diseño conceptual de una estructura reticular de cubierta con superficie de forma libre y geometría aleatoria de apoyos. Esto implica que el proceso morfogenético de optimización debe ser capaz de diseñar este tipo de estructura sin importar la forma de la planta del espacio, ni la posición de los apoyos. El objetivo de la optimización es minimizar la energía de deformación y el peso de la estructura, mientras que las definiciones que se mantendrán constantes durante el proceso serán las coordenadas $x-y$ de los nodos de la estructura reticular, el área de la sección transversal de las barras y el material de éstas. De esta manera, la variable será la coordenada $z$ de los nodos de la estructura reticular. A estas variables, se les aplican unas restricciones con el fin de forzarlas dentro de un espacio geométrico determinado, definido por una altura deseada mínima y máxima. Como resultado, debe ser conducido un proceso morfogenético de diseño, en el cual el algoritmo de optimización debe cambiar la posición $z$ de los nodos con el fin de encontrar una configuración geométrica óptima o sub-óptima (correspondiente a las funciones objetivo pre-definidas) para la estructura reticular de cubierta.

\section{PROCESO DE MORFOGÉNESIS}

El proceso de morfogénesis está basado en la integración de un procedimiento automático de generación de geometría llevado a cabo en Rhinoceros (2013) + Grasshopper (2013) + LunchBox (2013), con un código de programación para el análisis estructural (basado en el Método de Elementos Finitos, FEM) dentro de un algoritmo heurístico, el UPSO (Parsopoulos y Vrahatis, 2004) de optimización multi-objetiva. 


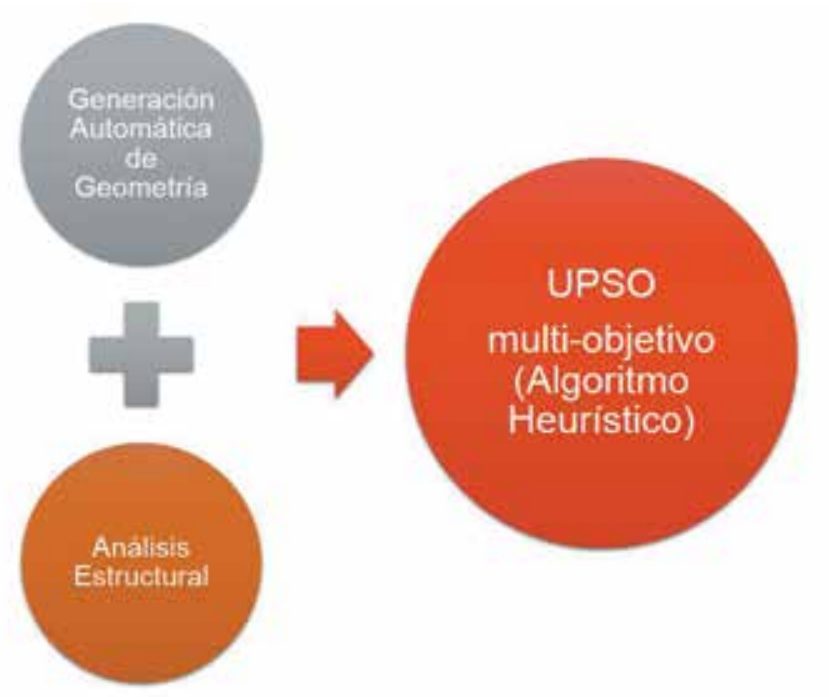

Figura I. Componentes del proceso de optimización

Fuente: Elaboración de los autores.

\section{Algoritmo heurístico - UPSO multi-objetivo}

EI UPSO es un acercamiento que combina las capacidades de exploración y explotación del algoritmo Particle Swarm Optimization, PSO (Kennedy y Eberhart, 1995). Para esto, la velocidad de las partículas (posibles soluciones) es actualizada en base a un variante global, $\mathcal{G}_{i}^{(k+1)}$ y a uno local, $\mathcal{L}_{i}^{(k+1)}$, del PSO (Parsopoulos y Vrahatis, 2004):

$$
\begin{aligned}
& \mathcal{G}_{i}^{(k+1)}=x\left[v_{i}^{(k)}+c_{1} r_{1}\left(p_{i}^{(k)}-x_{i}^{(k)}\right)+c_{2} r_{2}\left(p_{g}^{(k)}-x_{i}^{(k)}\right)\right] \\
& \mathcal{L}_{i}^{(k+1)}=x\left[v_{i}^{(k)}+c_{1} r_{1}^{\prime}\left(p_{i}^{(k)}-x_{i}^{(k)}\right)+c_{2} r_{2}^{\prime}\left(p_{g i}^{(k)}-x_{i}^{(k)}\right)\right]
\end{aligned}
$$

Donde $i$ es el número de la partícula (posible solución); $k$ es el número de la iteración; $x$ es el factor de constricción (un parámetro que controla la magnitud de la velocidad); $v_{i}^{(k)}$ es la velocidad de la partícula $i$ - ésima en la iteración; $k ; p_{i}^{(k)}$ es la mejor posición que la partícula $i$ - ésima ha encontrado hasta la iteración; $k ; x_{i}^{(k)}$ es la posición de la partícula $i$ - ésima en la iteración; $k ; c_{1}$ y $c_{2}$ son dos constantes positivas (son parámetros de aceleración llamados, respectivamente, cognitivo y social); $r_{1}$ y $r_{2}$ son dos números aleatorios contenidos en el intervalo $[0,1] ; p_{g}^{(k)}$ es la mejor posición que ha encontrado la totalidad del enjambre hasta la iteración $k$ (variante global); $y p_{g i}^{(k)}$ es la mejor posición que ha encontrado el vecindario de $x_{i}^{(k)}$ hasta la iteración $k$ (variante local).

Luego, la actualización de la velocidad de cada partícula es una combinación de las ecuaciones (I) y (2) junto con un parámetro $u$, llamado el factor de unificación (con el fin de determinar la influencia que tienen los componentes globales y locales); si $u=1$, la actualización de la velocidad es equivalente al variante global del PSO, mientras que si $u=0$, será equivalente al variante local del PSO (Parsopoulos y Vrahatis, 2004):

$$
U_{i}^{(k+1)}=u \mathcal{G}_{i}^{(k+1)}+(1-u) \mathcal{L}_{i}^{(k+1)}
$$

Finalmente, la actualización de la posición de la partícula estará determinada por (Parsopoulos y Vrahatis, 2004):

$$
x_{i}^{(k+1)}=x_{i}^{(k)}+U_{i}^{(k+1)}
$$


Adicionalmente, se propone aplicar el acercamiento Conventional Weighted Aggregation, CWA (Parsopoulos y Vrahatis, 2002a) para manejar la multiplicidad de objetivos. En este acercamiento, todos los objetivos $f_{i}(x)$, se suman en una combinación con pesos fijos de la siguiente manera:

$$
F=\sum_{i=1}^{k} w_{i} f_{i}(x)
$$

Donde $w_{i}$, con $i=1, \ldots, k$, son pesos no negativos; usualmente se asume que $\sum_{i=1}^{k} w_{i}=1$.
Luego, la función a optimizar sería $F$.

En cuanto a la aplicación de restricciones, éstas se manejaron por medio del acercamiento de función de penalización (Parsopoulos y Vrahatis, 2002b). Este acercamiento se fundamenta en redefinir la función objetivo, en este caso $F$, por medio de la inclusión de una función que penaliza, proporcionalmente, las soluciones que incumplen con las restricciones impuestas; entre más distante esté la solución del cumplimiento de la restricción, el valor de penalización será mayor. Para llevar a cabo este acercamiento, es necesario definir las restricciones como se muestra a continuación:

$$
g_{i}(x) \leq 0, \quad i=1, \ldots, m
$$

Donde $i$ hace referencia al número de la restricción. Si la restricción está planteada como $g_{i}(x) \geq 0$, se debe replantear como $-g_{i}(x) \leq 0$; en cambio, si la restricción es de igualdad $g_{i}(x)=0$, debe replantearse como dos restricciones: $g_{i}(x) \leq 0$ y $-g_{i}(x) \leq 0$.

Luego de definir las restricciones, se replantea la función objetivo así:

$$
F(x)=f(x)+h(k) H(x), \quad x \in S \subset \mathbb{R}^{n}
$$

Donde $f(x)$ es la función objetivo original (sin restricciones); $h(k)$ es un valor de penalización dinámicamente modificado, siendo $k$ el número de la iteración actual del algoritmo; y $H(x)$ es el factor de penalización, definido por:

$$
H(x)=\sum_{i=1}^{m} \theta\left(q_{i}(x)\right) q_{i}(x)^{\gamma\left(q_{i}(x)\right)}
$$

Donde, $q_{i}(x)=\max \left\{0, g_{i}(x)\right\}, i=1, \ldots, m$. La función $q_{i}(x)$ es una función de violación relativa de las restricciones; $\theta\left(q_{i}(x)\right)$ es una función de asignación multi-fase; $y \gamma\left(q_{i}(x)\right)$ es la potencia de la función de penalización.

Para la definición de cada una de las anteriores funciones, se planteó lo expuesto por Parsopoulos y Vrahatis (2002a). En este sentido, dichas funciones se fijaron de la siguiente forma:

$$
\begin{array}{r}
\gamma\left(q_{i}(x)\right)=\left\{\begin{array}{l}
1 \text { si } q_{i}(x)<1 \\
2 \text { si } q_{i}(x) \geq 1
\end{array}\right. \\
\theta\left(q_{i}(x)\right)=\left\{\begin{array}{l}
10 \text { si } q_{i}(x)<0.001 \\
20 \text { si } q_{i}(x) \leq 0.100 \\
100 \text { si } q_{i}(x) \leq 1.000 \\
300 \text { si } q_{i}(x)>1.000
\end{array}\right. \\
h(k)=\sqrt{k} \quad \text { (II })
\end{array}
$$


Finalmente como resultado, el acercamiento propuesto de optimización es un UPSO Multi-Objetivo con Restricciones (CMOUPSO por sus siglas en inglés, Constrained MultiObjective UPSO).

\section{Generación automática de geometría}

La geometría inicial de la estructura reticular de cubierta es generada con Grasshopper + LunchBox, un plug-in de Rhinoceros y un plug-in de Grasshopper, respectivamente. Con estos plug-ins, es posible programar la creación de los nodos de la estructura y la conectividad de Delaunay (De Berg, 2008) entre ellos (la cual definirá las conexiones de los elementos de la estructura), necesitando como datos de entrada, únicamente las coordenadas de los puntos de apoyo y el número deseado de divisiones en el plano $x-y$ (con el fin de crear la retícula de la estructura). En el desarrollo de este proceso, también es necesario el uso de MatLab para generar aleatoriamente la coordenada $z$ de cada uno de los nodos (estos valores serán las variables durante el proceso de optimización) y para filtrar y organizar la información de todos ellos.

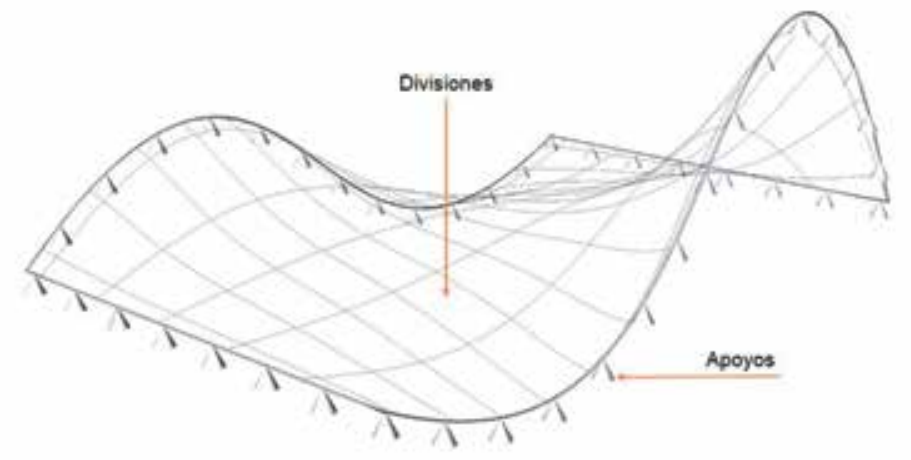

Consecuentemente, el resultado de este proceso es una tabla o matriz (una por partícula del CMOUPSO), guardada en MatLab, con los nodos ordenados, sus coordenadas, y la información de la conectividad de Delaunay. Estos datos serán utilizados durante el proceso de optimización multi-objetiva para el análisis estructural y el cálculo del peso.

\section{Análisis estructural}

En este caso, el propósito del análisis estructural es calcular la energía de deformación de cada estructura reticular de cubierta (correspondiente a cada partícula del CMOUPSO) con el fin de minimizarla durante el proceso de optimización multi-objetiva. Para esto, se programó en MatLab el FEM, siguiendo los planteamientos presentados por Chandrupatla y Belegundu (20I I) para elementos sometidos a cargas axiales. Finalmente, la energía de deformación se calculó por medio de la ecuación 5 , en la cual se fundamenta la primera función objetivo:

$$
E_{S}=(\{D\}[K])\{D\}^{\prime}
$$

Figura 2. Ejemplo de la geometría de entrada necesaria para Grasshopper (puntos de apoyo y número de divisiones para la retícula) Fuente: Elaboración de los autores. 
Donde $\{D\}$ es el vector de desplazamientos; $[K]$ la matriz de rigidez de la estructura reticular; y $\{D\}^{\prime}$ es la transpuesta de $\{D\}$. Esta ecuación también puede verse así:

$$
E_{S}=\{F\}\{D\}^{\prime}
$$

Donde $\{F\}=\{D\}[K]$ es el vector de fuerzas nodales.

Otro valor que debe ser calculado es el peso de la estructura con el fin de minimizarlo. La información resultante del proceso automático de generación de geometría, es utilizada para obtener el volumen total de material utilizado en las barras de la estructura reticular. Luego, este valor es multiplicado por la densidad del acero y la aceleración de la gravedad, calculando así el peso total de la estructura, en el cual se fundamenta la segunda función objetivo:

$$
W=g * \rho_{\text {steel }} \sum_{i=1}^{n} V_{r_{i}}
$$

Donde $g$ es la aceleración de la gravedad; $\rho_{\text {steel }}$ es la densidad del acero; $n$ es el número de barras; $y V_{r_{i}}$ es el volumen de la $i$ - ésima barra.

\section{PROBLEMAS DE PRUEBA Y RESULTADOS}

Para presentar el funcionamiento del proceso de morfogénesis, se plantearon dos problemas de prueba con el objetivo de buscar la mejor posición (sobre el eje z) de cada nodo y así lograr minimizar la energía de deformación (objetivo No. I, ver ecuación (I2)) y el peso de la estructura (objetivo No. 2, ver ecuación (14)).

El primer problema hace referencia a una estructura reticular de cubierta con un contorno regular de apoyos (todos con la misma altura y una forma geométrica regular), correspondiente a una planta rectangular de $5.00 \times 8.00 \mathrm{~m}$, con 119 barras y 52 nodos (ver Figura 3). Con este ejemplo, se pretende mostrar la convergencia del algoritmo, así como los resultados formales del proceso de morfogénesis con sus respectivas disminuciones en el peso y en la energía de deformación. El segundo ejemplo, corresponde a la aplicación del proceso de morfogénesis sobre una estructura reticular de cubierta con una forma en planta y un contorno de apoyos definidos por un octágono circunscrito en un círculo de $6.00 \mathrm{~m}$ de diámetro (ver Figura 4). En este caso, la estructura está compuesta por 31 nodos y 77 barras.

En cuanto a las definiciones necesarias para llevar a cabo el análisis estructural y el cálculo del peso, en los dos ejemplos la sección transversal de las barras es circular, con un área de $126.7 \mathrm{~mm} 2$ (correspondiente a un diámetro de); el material de las barras es acero con módulo de elasticidad de $200000 \mathrm{MPa}$ y densidad de $7800 \mathrm{~kg} / \mathrm{m}^{3}$. La carga aplicada, en los dos casos, fue de $1000 \mathrm{~N}$ en dirección de la gravedad, en cada uno de los nodos de la estructura.

Por otro lado, las restricciones aplicadas a la coordenada $\mathrm{z}$ de cada nodo (las variables durante el proceso de morfogénesis), se plantearon para delimitar un rango espacial, i.e. una altura máxima y una mínima. En los dos ejemplos, esta restricción se definió en función de las dimensiones en planta, para que el resultado formal final tuviera la libertad de formar tipologías de bóvedas o cúpulas (dejando un margen de movilidad considerable). 


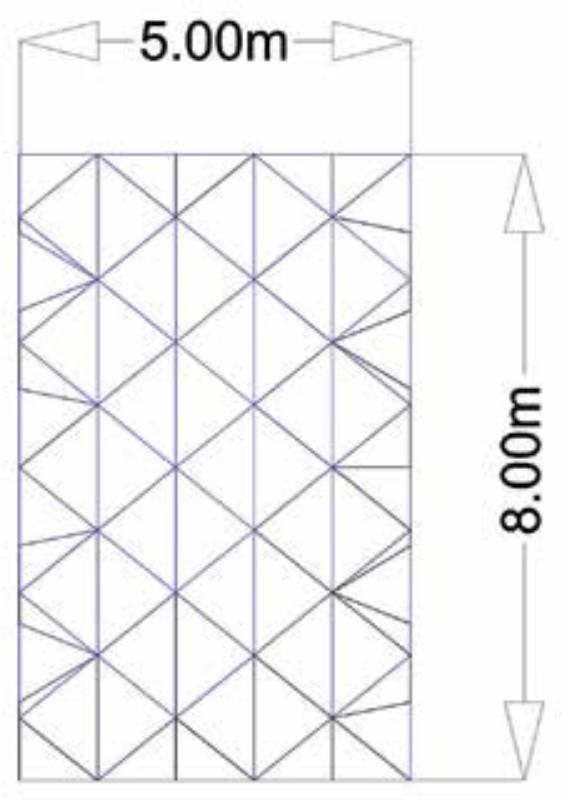

Figura 3. Planta de la estructura reticular de cubierta correspondiente al problema de prueba I

Fuente: Elaboración de los autores.

Figura 4. Planta de la estructura reticular

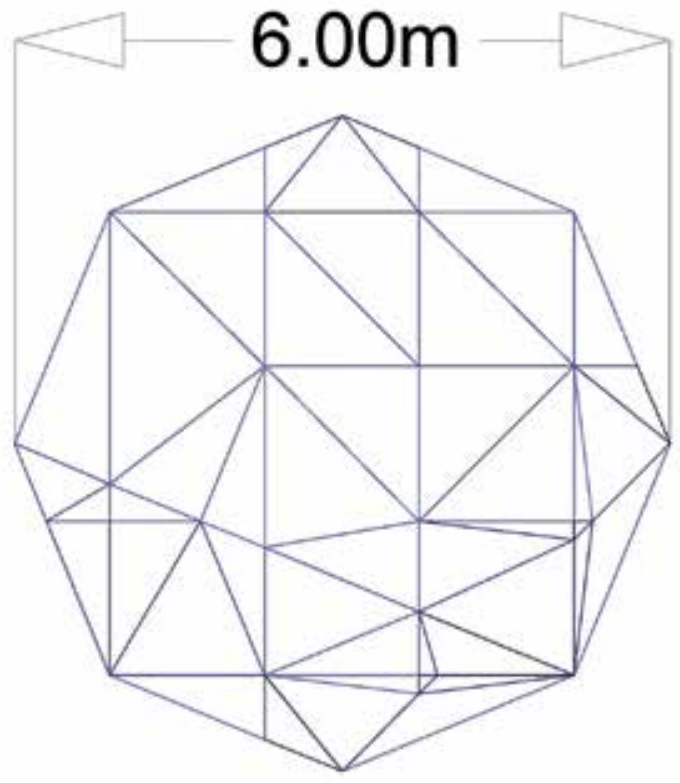

de cubierta correspondiente al problema de prueba 2

Fuente: Elaboración de los autores.

\section{Resultados del problema de prueba I}

El primer problema de prueba tuvo dos fases, la primera para mostrar la convergencia del algoritmo, y la segunda para mostrar la variación formal correspondiente a distintas combinaciones de peso. Para la primera fase, el algoritmo se corrió 5 veces, con 1000 partículas, durante 50 iteraciones y con una combinación de pesos en donde $\mathrm{wl}=0.5$ 
(correspondiente al objetivo de energía de deformación) y w2 $=0.5$ (correspondiente al objetivo de peso); los parámetros utilizados fueron los siguientes:

- $c_{1}=1.4$

- $c_{2}=1.6$

- $X=0.5$

- $u=0.7$

Los resultados de esta fase son los que se muestran en la Tabla I y en las Figuras 5, 6 y 7. A partir de ellos, es posible comprobar la convergencia del algoritmo (la convergencia correspondiente a la energía de deformación se puede ver específicamente en la Figura 8, en donde se evidencia que el algoritmo converge a partir de la iteración No. 40), debido a que los resultados tendieron a ser semejantes en las distintas corridas. Por ejemplo, la desviación estándar en el valor del peso mínimo encontrado al final del proceso fue de $50.17 \mathrm{~kg}$, tan sólo un $2.82 \%$ del promedio de dicho objetivo (el promedio del peso fue de $1780.78 \mathrm{~kg}$ ). Por otro lado, la desviación estándar en el valor de la energía de deformación mínima encontrada por el algoritmo, fue de $50.88 \mathrm{~N} * \mathrm{~mm}$, alcanzando un porcentaje de $2.19 \%$ del promedio de estos valores (el promedio de la energía de deformación fue de $2324.73 \mathrm{~N} * \mathrm{~mm})$.

Tabla I. Resultados de la optimización de peso y energía de deformación, correspondientes a 5 corridas distintas del proceso de morfogénesis (con pesos $w I=0.5$ y w2 $=0.5$ ). Fuente: Elaboración de los autores.

\begin{tabular}{|c|c|c|c|c|c|c|c|}
\hline Corrida & $\begin{array}{c}\text { Peso } \\
\text { Inicial } \\
{[\mathbf{N}]}\end{array}$ & $\begin{array}{c}\text { Peso } \\
\text { Final } \\
{[\mathbf{N}]}\end{array}$ & $\begin{array}{c}\text { Diferencia } \\
{[\%]}\end{array}$ & $\begin{array}{c}\text { Energía de } \\
\text { Deformación } \\
\text { Inicial }\left[\mathbf{N}^{*} \mathbf{m m} \text { ] }\right.\end{array}$ & $\begin{array}{c}\text { Energía de } \\
\text { Deformación } \\
\text { Final [N*mm] }\end{array}$ & $\begin{array}{c}\text { Diferencia } \\
{[\%]}\end{array}$ & $\begin{array}{c}\text { Tiempo } \\
{[\mathbf{s e g}]}\end{array}$ \\
\hline 1 & 2941.23 & 1748.92 & -40.54 & 8727.72 & 2408.06 & -72.41 & 462.23 \\
\hline 2 & 2833.51 & 1708.39 & -39.71 & 7345.56 & 2283.40 & -68.91 & 473.31 \\
\hline 3 & 3028.63 & 1805.76 & -40.38 & 10006.77 & 2293.59 & -77.08 & 456.10 \\
\hline 4 & 2895.45 & 1825.24 & -36.96 & 17174.64 & 2337.66 & -86.39 & 452.83 \\
\hline 5 & 2999.99 & 1815.61 & -39.48 & 6701.14 & 2300.92 & -65.66 & 457.79 \\
\hline
\end{tabular}

Figura 5. Convergencia de la optimización del peso correspondiente al problema de prueba No. I

Fuente: Elaboración de los autores.

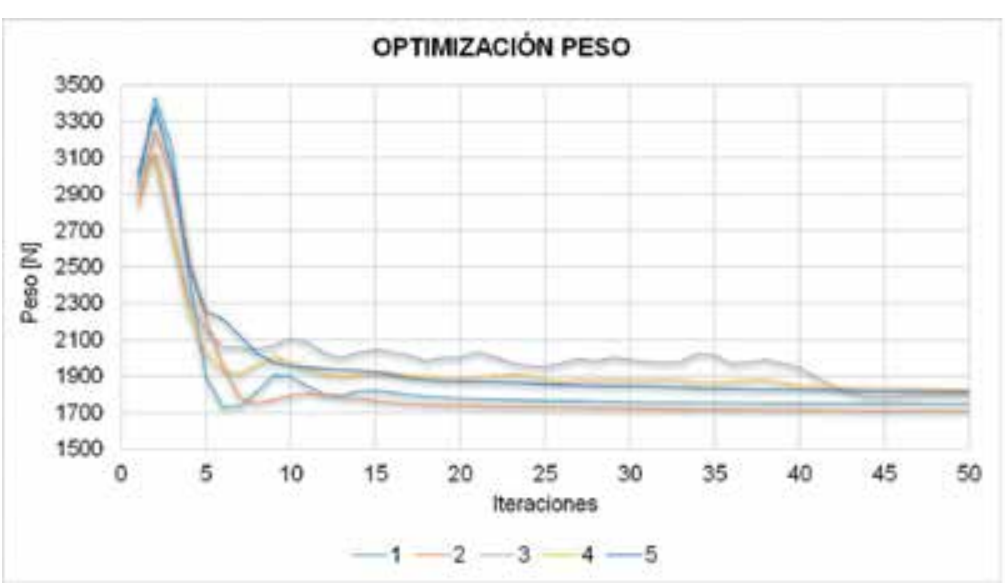



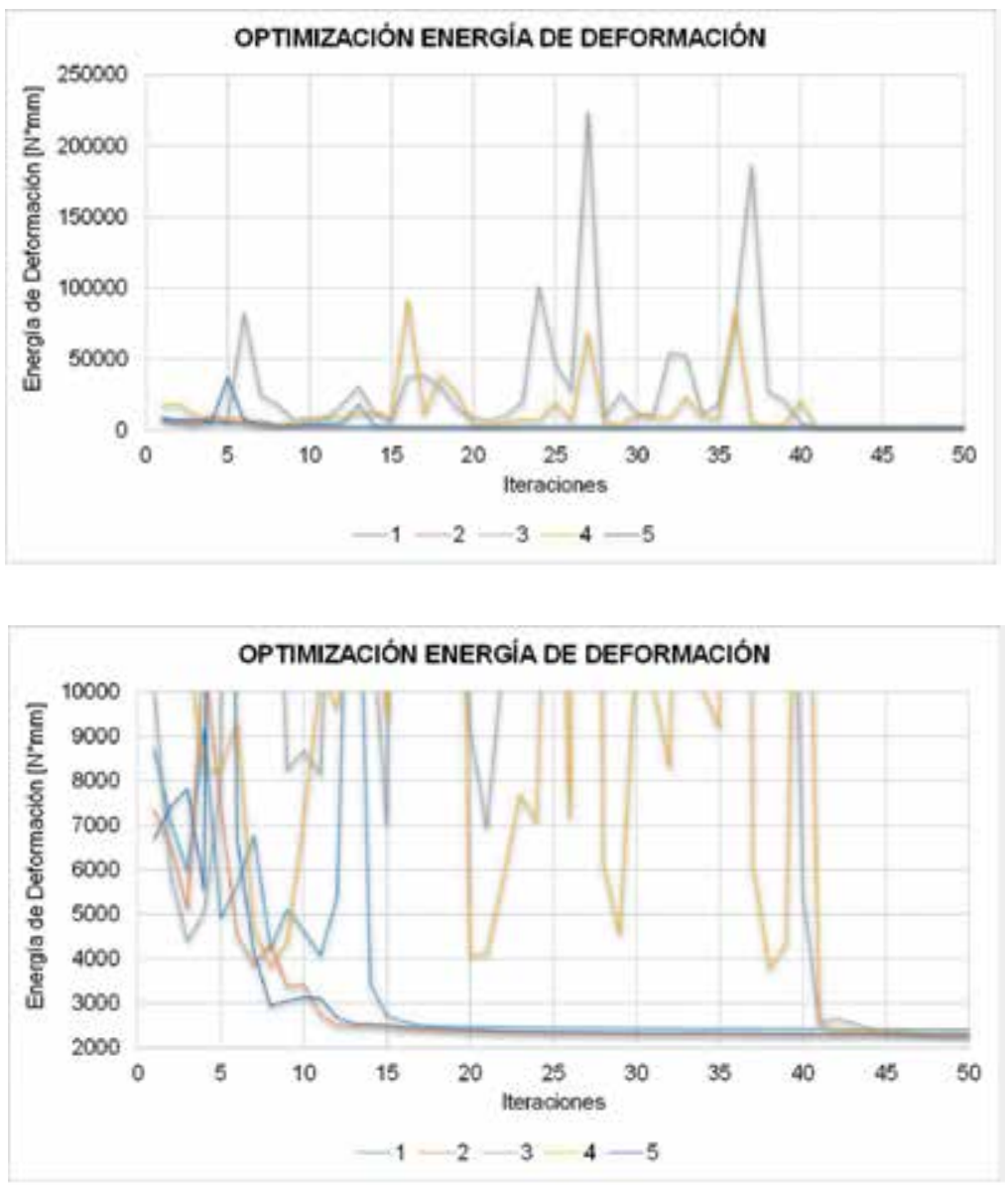

En cuanto al resultado formal de esta fase, todas las corridas del algoritmo tendieron a configurar una tipología estructural de domo, como se puede observar en las Figuras 8a, $8 b$ y $8 c$. Esta morfología corresponde con los resultados encontrados por Richardson, Adriaenssens, Coelho y Bouillard (2013) y al reconocimiento que se le ha dado a dicha tipología estructural, debido a su eficiencia, a lo largo de la historia: desde el Panteón romano, pasando por la basílica de Santa Maria del Fiore, y llegando a ejemplos más recientes como el Eden Project.

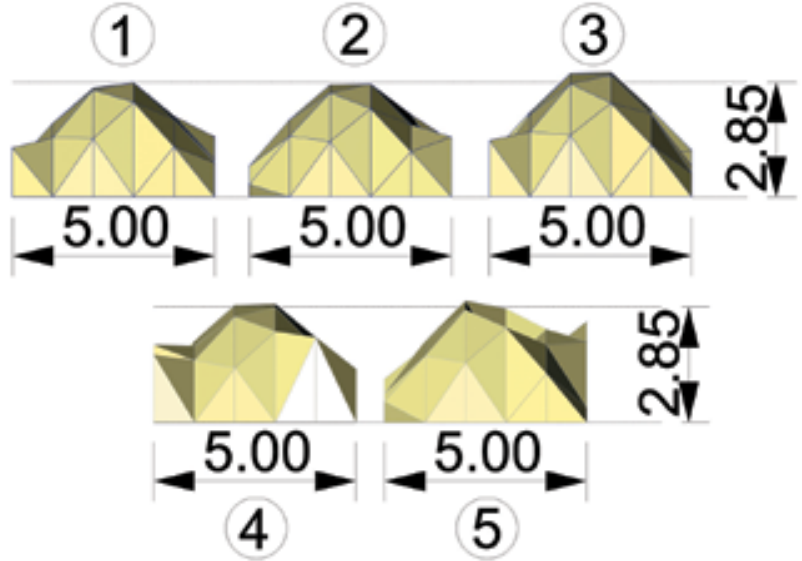

Figura 6. Convergencia de la optimización de la energía de deformación correspondiente al problema de prueba No. I Fuente: Elaboración de los autores.

Figura 7. Acercamiento de la Figura 6 Fuente: Elaboración de los autores.

Figura 8a. Vista frontal de la cubiertas finales, correspondientes a 5 corridas distintas del proceso de morfogénesis, con pesos $w I=0.5 y$ $w 2=0.5$ (ver Tabla I).

Fuente: Elaboración de los autores. 
Figura 8b. Vista lateral de la cubiertas finales, correspondientes a 5 corridas distintas del proceso de morfogénesis, con pesos $w I=0.5 y$ w2 $=0.5$ (ver Tabla I).

Fuente: Elaboración de los autores.
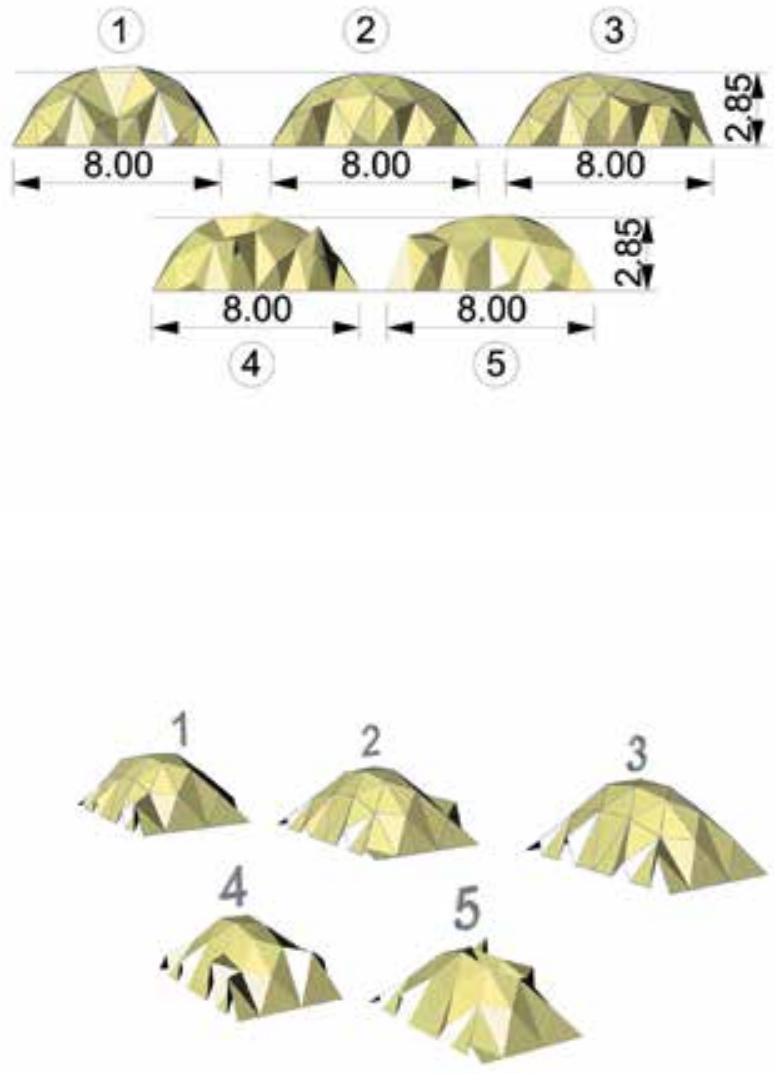

Para el desarrollo de la segunda fase, el algoritmo, definido con los mismos parámetros de la fase I, se corrió con 5 combinaciones de pesos distintas (en donde $\mathrm{wl}$ corresponde al objetivo de la energía de deformación y $w 2$ al de peso): $w \mathrm{l}=0.10$ y $w 2=0.9, \mathrm{wl}=0.30$ y $w 2=0.7, w I=0.50$ y $w 2=0.5, w I=0.70$ y $w 2=0.3, w I=0.90$ y $w 2=0.1$. Los resultados de este proceso son los que se muestran en la Tabla 2 y en la Figura 9, en donde es posible observar la generación de una aproximación al frente de Pareto. Adicionalmente, las soluciones allí incluidas presentaron una reducción promedio en el peso de $43.88 \%$ en comparación con las estructuras iniciales generadas aleatoriamente, mientras que se logró reducir la energía de deformación, en promedio un $68.62 \%$. Otro dato importante a tener en cuenta es el tiempo que se demoró el algoritmo en llevar a cabo el proceso que, para este caso, fue de 474.54 segundos en promedio.

\begin{tabular}{|c|c|c|c|c|c|c|c|c|}
\hline \multicolumn{2}{|c|}{ Pesos } & \multirow{2}{*}{$\begin{array}{l}\text { Peso } \\
\text { Inicial } \\
\text { [N] }\end{array}$} & \multirow{2}{*}{$\begin{array}{c}\text { Peso } \\
\text { Final } \\
{[\mathrm{N}]}\end{array}$} & \multirow{2}{*}{$\begin{array}{c}\text { Diferencia } \\
\text { [\%] }\end{array}$} & \multirow{2}{*}{$\begin{array}{c}\text { Energía de } \\
\text { Deformación } \\
\text { Inicial }\left[\mathrm{N}^{*} \mathrm{~mm}\right]\end{array}$} & \multirow{2}{*}{$\begin{array}{c}\text { Energía de } \\
\text { Deformación } \\
\text { Final }\left[\mathrm{N}^{\star} \mathrm{mm}\right]\end{array}$} & \multirow{2}{*}{$\begin{array}{c}\text { Diferencia } \\
{[\%]}\end{array}$} & \multirow{2}{*}{$\begin{array}{c}\text { Tiempo } \\
\text { [seg] }\end{array}$} \\
\hline w1 & w2 & & & & & & & \\
\hline 0.10 & 0.90 & 3169.49 & 1527.93 & -51.79 & 7079.14 & 3561.52 & -49.69 & 462.48 \\
\hline 0.30 & 0.70 & 2955.79 & 1601.30 & -45.82 & 16088.35 & 2960.55 & -81.60 & 473.89 \\
\hline 0.50 & 0.50 & 2828.83 & 1737.07 & -38.59 & 8607.15 & 2642.18 & -69.30 & 478.62 \\
\hline 0.70 & 0.30 & 3215.59 & 1849.43 & -42.49 & 9476.46 & 2416.47 & -74.50 & 484.36 \\
\hline 0.90 & 0.10 & 3164.25 & 1875.60 & -40.73 & 7131.78 & 2281.23 & -68.01 & 473.37 \\
\hline
\end{tabular}

Tabla 2. Resultados de la optimización de peso y energía de deformación, correspondientes a 5 combinaciones distintas de pesos. Problema de prueba No. I

Fuente: Elaboración de los autores.
. Perspectiva de la cubiertas finales, $w 2=0.5$ (ver Tabla I). 


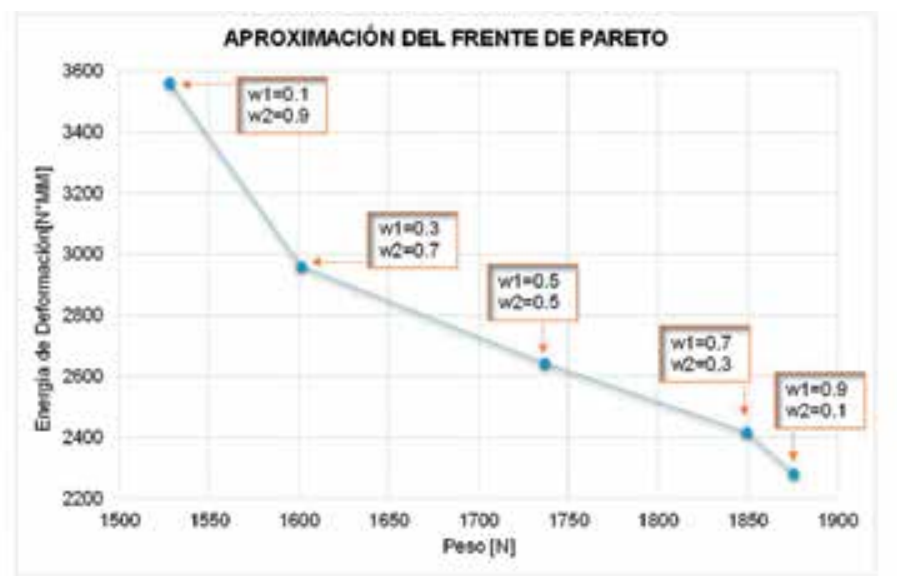

Finalmente, en cuanto al resultado formal de las estructuras, es posible observar en las Figuras 10a, 10b y 10c, la variación en la configuración geométrica de la estructura, correspondiente a las distintas combinaciones de pesos. Cuando la combinación de pesos le da mayor prioridad al objetivo de minimización de peso, la cubierta tiende a ser más plana (cubiertas correspondientes a $\mathrm{wl}=0.1-\mathrm{w} 2=0.9 \mathrm{y} \mathrm{wl}=0.3-\mathrm{w} 2=0.7$, en las Figuras $10 \mathrm{a}$, IOb y 10c), mientras que si la prioridad está enfocada en la minimización de la energía de deformación, resulta en cubiertas con una altura mayor (cubiertas correspondientes a $w I=0.7-w 2=0.3$ y wl $=0.9-w 2=0.1$, en las Figuras $10 a, 10 b$ y $10 c$ ). Para todos los casos, el resultado formal se asemeja de nuevo a la tipología estructural de un domo.
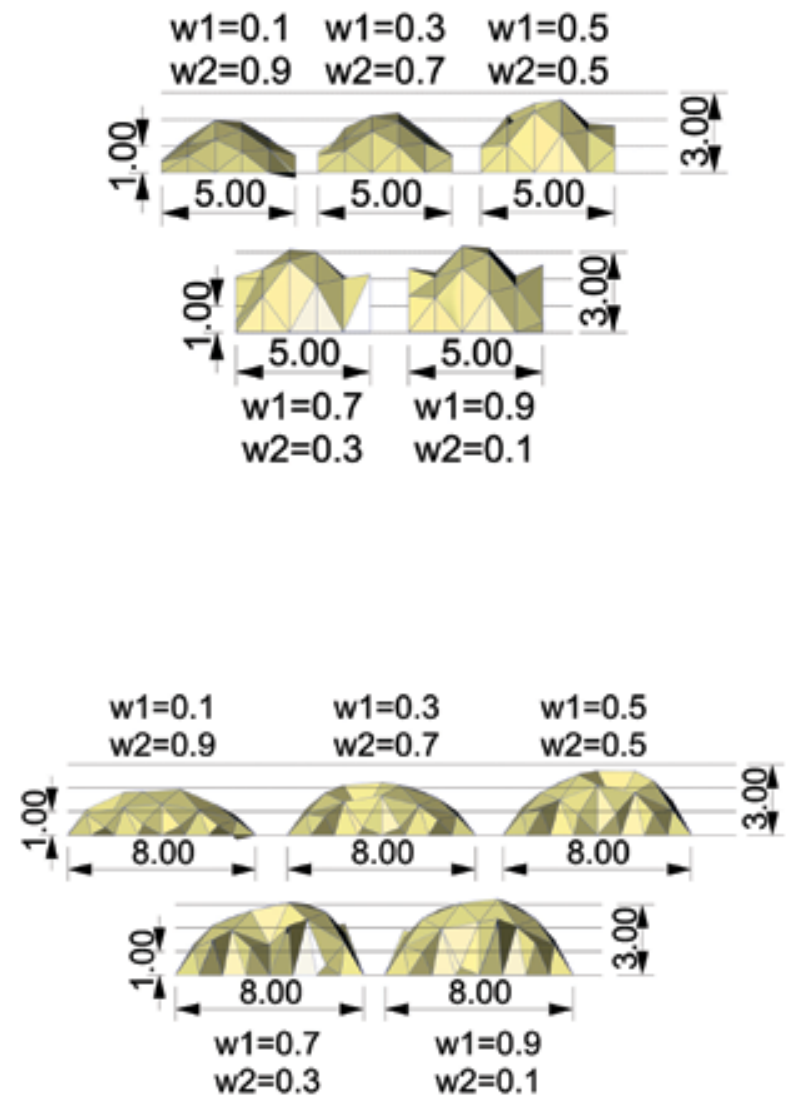

Figura 9. Aproximación del frente de Pareto para el problema de prueba No. I (ver Tabla 2) Fuente: Elaboración de los autores.

Figura 10a. Vista frontal de las cubiertas finales, correspondientes a 5 combinaciones distintas de pesos - Problema de prueba No. 1 (ver Tabla 2 y Figura 9). Fuente: Elaboración de los autores.
Figura I0b. Vista lateral de las cubiertas finales, correspondientes a 5 combinaciones distintas de pesos - Problema de prueba No. 1 (ver Tabla 2 y Figura 9)

Fuente: Elaboración de los autores. 
Figura 10c. Perspectiva de las cubiertas finales, correspondientes a 5 combinaciones distintas de pesos - Problema de prueba No. I (ver Tabla 2 y Figura 9)

Fuente: Elaboración de los autores.
Tabla 3. Resultados de la optimización de pe y energía de deformación correspondientes a 5 combinaciones distintas de pesos. Problema de prueba No. 2

Fuente: Elaboración de los autores.

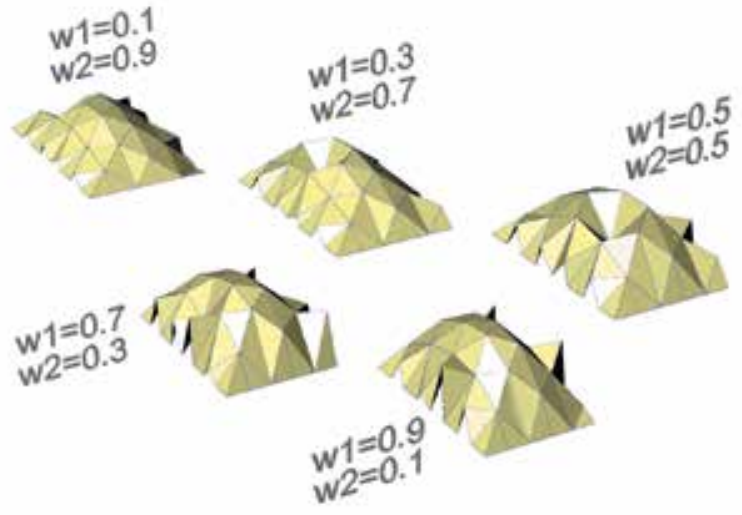

\section{Resultados del problema de prueba 2}

Para el segundo problema de prueba, el algoritmo corrió con la misma definición de parámetros del problema de prueba $\mathrm{I}$, con 1000 partículas, durante 50 iteraciones, y con 5 combinaciones distintas de pesos: (en donde $\mathrm{wl}$ corresponde al objetivo de la energía de deformación y $w 2$ al de peso): $w I=0.10$ y $w 2=0.9, w I=0.30$ y $w 2=0.7, w I=0.50$ y $\mathrm{w} 2=0.5, \mathrm{wl}=0.70$ y $\mathrm{w} 2=0.3, \mathrm{wl}=0.90$ y w2 $=0.1$. Los resultados obtenidos son los que se muestran en la Tabla 3 y la Figura II, en donde es posible observar la aproximación del frente de Pareto y una reducción del peso y de la energía de deformación en promedio de $34.51 \%$ y $56.11 \%$ respectivamente; los anteriores valores se obtienen en relación a las estructuras iniciales generadas aleatoriamente. En cuanto al tiempo promedio que se demoró el algoritmo en ejecutar el proceso de morfogénesis y optimización, en este caso fue de 329.33 segundos.

\begin{tabular}{|l|c|c|c|c|c|c|c|c|}
\hline \multicolumn{2}{|c|}{ Pesos } & $\begin{array}{c}\text { Peso } \\
\text { Inicial } \\
{[\mathrm{N}]}\end{array}$ & $\begin{array}{c}\text { Peso } \\
\text { Final } \\
{[\mathrm{N}]}\end{array}$ & $\begin{array}{c}\text { Diferencia } \\
{[\%]}\end{array}$ & $\begin{array}{c}\text { Energia de } \\
\text { Deformación } \\
\text { Inicial [N*mm] }\end{array}$ & $\begin{array}{c}\text { Energia de } \\
\text { Deformación } \\
\text { Final [N*mm] }\end{array}$ & $\begin{array}{c}\text { Diferencia } \\
{[\%]}\end{array}$ & $\begin{array}{c}\text { Tiempo } \\
\text { [seg] }\end{array}$ \\
\hline w1 & w2 & & & & & & \\
\hline 0.10 & 0.90 & 1540.93 & 1016.03 & -34.06 & 8206.57 & 5866.45 & -28.52 & 320.73 \\
\hline 0.30 & 0.70 & 1864.43 & 1120.18 & -39.92 & 5370.13 & 3795.17 & -29.33 & 331.41 \\
\hline 0.50 & 0.50 & 1737.11 & 1150.74 & -33.76 & 37309.81 & 3447.10 & -90.76 & 335.58 \\
\hline 0.70 & 0.30 & 1954.10 & 1242.91 & -36.39 & 14520.83 & 3293.44 & -77.32 & 352.03 \\
\hline 0.90 & 0.10 & 1805.93 & 1292.59 & -28.43 & 7094.59 & 3220.53 & -54.61 & 306.91 \\
\hline
\end{tabular}

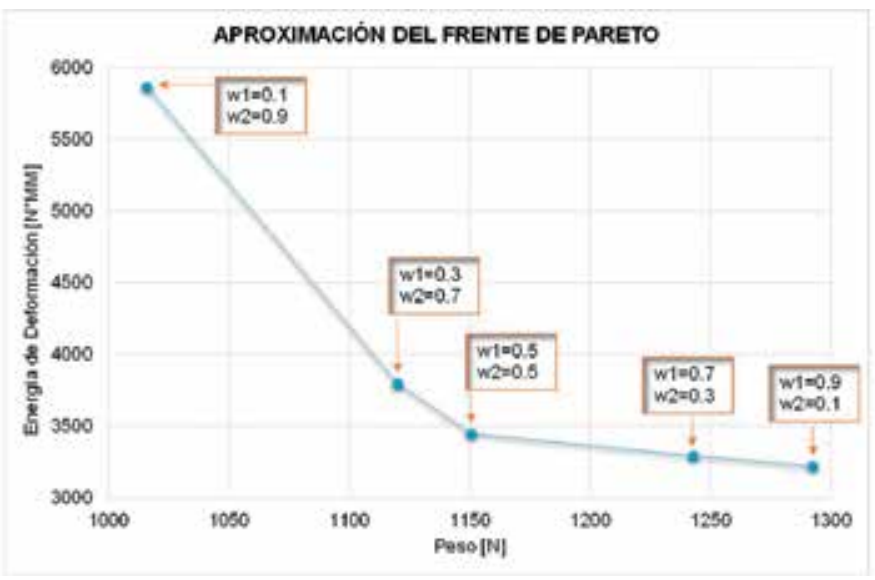

Figura II . Aproximación del frente de Pareto para el problema de prueba No. 2 (ver Tabla 3) Fuente: Elaboración de los autores. 
Por último, el resultado formal de las cubiertas del problema de prueba No. 2, se asemeja, de igual forma, a una tipología estructural tipo domo (ver Figuras I2a, I2b y I2c). Al igual que en el problema de prueba No. I, cuando la prioridad en el proceso de optimización es la minimización del peso, las cubiertas tienden a ser más planas (esto debido a que el peso depende directamente de la longitud de las barras), mientras que, cuando la prioridad es la minimización de la energía de deformación, el domo que define a la cubierta tiende a ser más profundo.
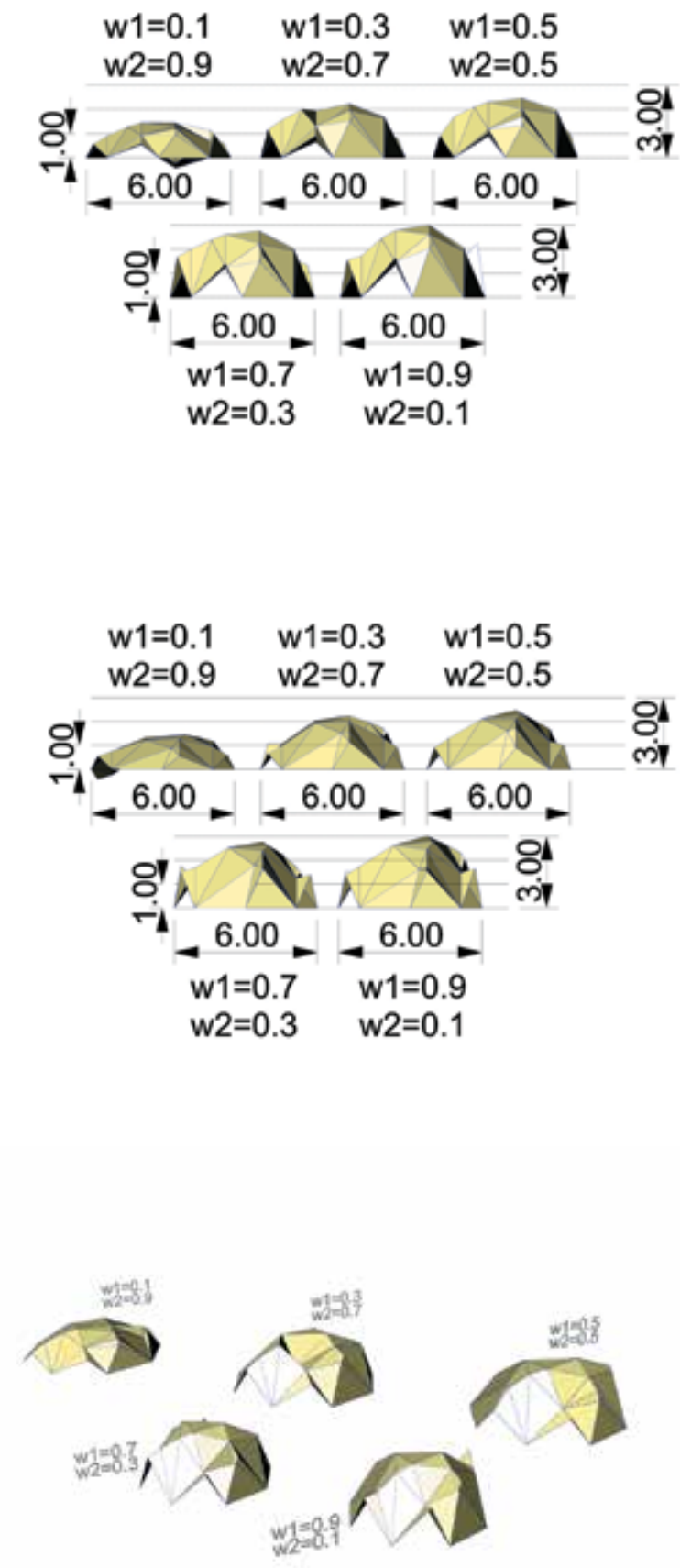

Figura 12a. Vista frontal de las cubiertas finales, correspondientes a 5 combinaciones distintas de pesos - Problema de prueba No. 2 (ver Tabla 3 y Figura II)

Figura I 2b. Vista lateral de las cubiertas finales, correspondientes a 5 combinaciones distintas de pesos - Problema de prueba No. 2 (ver Tabla 3 y Figura II)
Figura I2c. Perspectiva de las cubiertas finales, correspondientes a 5 combinaciones distintas de pesos - Problema de prueba No. 2 (ver Tabla 3 y Figura II) 


\section{CONCLUSIONES}

En esta investigación se propuso un procedimiento morfogenético de optimización multiobjetiva, el cual es capaz de generar y encontrar una aproximación de la mejor configuración geométrica para estructuras reticulares de cubierta, y que sea correspondiente a la minimización de la energía de deformación y el peso. Por medio de los problemas de prueba presentados, fue posible demostrar la eficiencia de dicho procedimiento, debido a que, sin importar la forma de la planta que cubre la estructura reticular, se obtuvieron reducciones en el peso entre un $28 \%$ y un $52 \%$, mientras que la reducción en la energía de deformación osciló entre un $28 \%$ y un $91 \%$ (correspondientes a la comparación con las estructuras iniciales generadas aleatoriamente). Aparte de estos resultados técnicos, el procedimiento permite que el diseñador pueda variar los pesos (la importancia) asignados a cada objetivo (energía de deformación y peso), obteniendo resultados formales distintos. De esta manera, se garantiza un alto rendimiento (en función de los objetivos deseados) y se permiten distintas variaciones geométricas para, posteriormente, seleccionar una de ellas. Consecuentemente, el resultado final será un diseño conceptual técnicamente óptimo y estéticamente agradable (para quien lleve a cabo el diseño).

Como pasos futuros en este campo de investigación, se propone la inclusión de una mayor cantidad de objetivos que no sólo se limiten a la optimización estructural, sino que incluyan otras funciones que podrían estar enfocadas, por ejemplo, en el diseño bioclimático, sostenible, acústico, de iluminación, y, por qué no, urbanístico. Adicionalmente, se hace necesario el desarrollo de una herramienta que permita la interacción entre el diseñador y el procedimiento, con el fin de controlar, paso a paso, el resultado estético de las soluciones. De seguir este camino, se cree que la posibilidad de aproximarse a una solución holística, arquitectónica e ingenieril, podría ser una realidad.

\section{REFERENCIAS}

Byrne, J., Fenton, M., Hemberg, E., McDermott, J., O'Neil, M., Shotton, E. y Nally, C. (20II). Combining structural analysis and multi-objective criteria for evolutionary architectural design. Applications of Evolutionary Computation, 204-213.

Chandrupatla, T. y Belegundu, A. (20I I). Introduction to finite elements in engineering. Upper Saddle River, Prentice Hall. 
De Berg, M. (2008). Computational Geometry: Algorithms and Applications. Berlín: Springer.

Grasshopper. (20/3) [en línea]. <http://www.grasshopper3d.com/> [citado el 8 de julio de 20I3].

Grasshopper: LunchBox. (2013) [en línea]. http://www.grasshopper3d.com/group/lunchbox [citado el 8 de julio de 2013].

Kennedy, J. y Eberhart, R. (1995). Particle Swarm Optimization. Proceedings of the 1995 IEEE International Conference on Neural Networks. E.E.U.U: Piscataway.

Parsopoulos, K. y Vrahatis, M.N. (2002a). Particle Swarm Optimization method in multiobjective problems. Proceedings ACM Symposium on Applied Computing (SAC 2002). Madrid, España.

Parsopoulos, K. y Vrahatis, M.N. (2002b). Particle Swarm optimization method for constrained optimization problems. Proceedings of the second Euro-International Symposium on Computational Intelligence. Košice, Eslovaquia.

Parsopoulos, K y Vrahatis, M.N. (2004). UPSO: A Unified Particle Swarm Optimization Scheme. In: Lecture Series on Computer and Computational Sciences, I, 868-873.

Pugnale, A. y Sassone, M. (2007). Morphogenesis and structural optimization of shell structures with the aid of a Genetic Algorithm. In: Journal of the International Association for Shell and Spatial Structures, 48, 16I-166.

Richardson, J.N., Adriaenssens, S., Coelho, R.F.y Bouillard, P. (2013). Coupled form-finding and grid optimization approach for single layer grid shells. In: Engineering Structures, 52, 230-239.

Rhinoceros. (2013) [en línea]. <http://www.rhino3d.com/> [citado el 8 de julio de 2013]. 